\title{
Towards the continuum limit of screening lengths with chiral fermions
}

\section{Rajiv V. Gavai* and Sourendu Gupta}

Department of Theoretical Physics, Tata Institute of Fundamental Research,

Homi Bhabha Road, Mumbai 400005, India

E-mail: gavaietifr.res.in sgupta@tifr.res.in

\section{Robert Lacaze}

Service de Physique Théorique, CEA Saclay,

F-91191 Gif-sur-Yvette Cedex, France

E-mail: Robert. Lacazedcea.fr

\begin{abstract}
We investigate mesonic screening correlators at $T=2 T_{c}$ using the overlap fermions in the quenched approximation, where $T_{c}$ is the QCD phase transition temperature. Using lattices with temporal extent up to 8 we found that both pseudoscalar and vector correlators exhibit a nice $\cosh$ behaviour, leading to a plateau behaviour in the local screening masses as a function of distance. The $\rho$ and $\pi$ masses so determined show very little variation with the lattice spacing $a$. This augurs well for the use of chiral fermions, and further suggests the small deviations of these masses from the ideal gas values are genuine effects of interactions.
\end{abstract}

XXIVth International Symposium on Lattice Field Theory

July 23-28, 2006

Tucson, Arizona, USA

\footnotetext{
* Speaker.
} 


\section{Introduction}

Lattice quantum chromodynamics (QCD) predicts a new phase, called Quark-Gluon Plasma (QGP), at high enough temperatures. Furthermore, it has contributed substantially in our understanding of this phase, providing most of the solid information we currently have about QGP. Nevertheless, several outstanding questions about the nature of QGP still remain. Since the very early days, it has been recognised that the bulk thermodynamic quantities, such as the energy density, cannot be explained by a straightforward weak coupling expansion. With more precise computations on the lattice as well as higher orders of perturbation theory, the problem became more and more acute. Modifications in form of certain resummations, non-perturbative schemes, and intuitive models were proposed to explain the discrepancy. Quark number susceptibilities provided an independent, mostly successful, check on these, giving the weak coupling picture a boost, at least for $T \geq 3-5 T_{c}$. On the other hand, the $J / \psi$ and $\eta_{c}$ mesons seem to survive up to a few $T_{c}$. This together with the results from the relativistic heavy ion collider at BNL, which suggest a very small viscosity, gave rise the picture of a strongly coupled QGP just above $T_{c}$. Recently, a strong evidence from lattice QCD simulations emerged [1], suggesting that the fermionic excitations of QGP behave like quarks already close to $T_{c}$. Thus an excitation carrying unit strangeness and $1 / 3$ baryon number also carries electric charge of - $1 / 3$, i.e., behaves like a strange quark.

Clearly for a complete picture to emerge, it seems prudent to add as many pieces of information as can be obtained. Static screening lengths, which we focus on in this work, constitute one such important clue. One studies the screening of currents in a medium to extract long-range information on its composition. For exciting mesons with specific quantum numbers from the vacuum, simplest forms of currents with those quantum numbers are chosen. They should exhibit deconfinement related changes above the QCD phase transition temperature $\left(T_{c}\right)$ [2], while yielding the known spectrum at low temperatures. Detailed studies have shown that this indeed does happen in the vector, and axial-vector channels: the screening above $T_{c}$ appears to be due to nearly non-interacting quark anti-quark pairs in the medium [3, †. However, the scalar and pseudo-scalar screening masses show more complicated behavior - strong deviations from the ideal Fermi gas, and a strong temperature dependence. This puzzling behavior is generic - it has been seen in quenched [5] and dynamical simulations with two [6] and four flavors [2, 3, 6, 7] of staggered quarks, as well as with Wilson quarks [8]. Staggered fermions have broken flavour symmetry on the lattice and, indeed, extrapolation to continuum limit in the quenched case gave hints [9] of the deviations being cut-off effects. However, the extraction of these masses both for the staggered and the Wilson case was difficult due to the complex behaviour of the correlators, which lead to the failure of the expected plateau in the effective distance dependent masses.

Since the number of pions and their nature is intimately related to the actually realized chiral symmetry on the lattice, one expects any good realization of chiral fermions on the lattice to provide better insight into the problem of screening lengths. Overlap fermions [10] have the advantage of preserving exact chiral symmetry on the lattice, i.e, at finite lattice spacing, for any number of flavours in contrast to the Wilson fermions which break all chiral symmetries or staggered fermions which do so only partially but at the expense of breaking flavour symmetry. In our earlier work [11], we showed the pion screening mass to be closer to the ideal gas value but still distinctly lower than rho screening mass. We employed then lattices with 4 temporal sites, $N_{t}=4$, and a conjugate 
gradient based method for the overlap-Dirac operator. For temperatures $1.25 \leq T / T_{c} \leq 2$, we observed a very mild temperature dependence of the ratio of screening mass and the temperature. In the current study, we address the issue of continuum limit of these screening masses by extending to $N_{t}=6$ and 8 . We also used lattices with long $z$ extent to investigate more carefully the lowest lying masses and the faster Zolotarev method for the overlap-Dirac operator. All computations were done at $T=2 T_{c}$, since any temperature in the range $1.25 T_{c}$ to $2 T_{c}$ could suffice in view of our earlier observation on the $N_{t}=4$ lattice.

\section{Simulation Details}

The massless overlap Dirac operator $(D)$ can be defined [12] in terms of the Wilson-Dirac operator $\left(D_{w}\right)$ for negative mass by the relation

$$
D=1+D_{w}\left(D_{w}^{\dagger} D_{w}\right)^{-1 / 2} .
$$

As in [11], we chose the negative mass in $D_{w}$ to 1.8 in this work as well. The corresponding operator for massive quarks is

$$
D(m a)=m a+(1-m a / 2) D,
$$

where $m$ is the bare quark mass, $a$ the lattice spacing, and $D$ is defined above. We used the usual quark propagator, $G(m a)=[1-D / 2] D^{-1}(m a)$. To compute this, one needs the inverse of the massive overlap $D$, of eq. (2.2), which, as is widely known, needs a nested series of two matrix inversions. At each step in the numerical inversion of $D$, one has to invert $\left(D_{w}^{\dagger} D_{w}\right)^{1 / 2}$. For the matrix $M=D_{w}^{\dagger} D_{w}$, and a given source vector $b$, we computed $y=M^{-1 / 2} b$ by using the Zolotarev algorithm [13]:

$$
M^{-1 / 2} b=\sum_{l=1}^{N_{\mathscr{G}}}\left(\frac{c_{l}}{M+d_{l}} b\right) .
$$

Here the coefficients $c_{l}$ and $d_{l}$ are computed with Jacobi elliptic functions for a chosen order of approximation $N_{\mathscr{O}}$ and a ratio $\kappa=\mu_{\max } / \mu_{\min }$ where $\mu_{\max }$ and $\mu_{\min }$ bound of the domain for which we apply the approximation. In our implementation of the algorithm [14], we first compute the lowest and highest eigenvalues of $M$ for a given required precision $\varepsilon$. Then we define the bound of the domain by increasing by $10 \%$ the domain of eigenvalues. The order $N_{\mathscr{O}}$ is defined by requiring a precision $\varepsilon / 2$ for the approximation of $1 / \sqrt{z}$ in the entire domain. We used $\varepsilon=10^{-5}$ and found that typically $N_{\mathscr{O}} \sim 7-8$ was needed. With these parameters, one calculates the approximation in eq. (2.3) by a multishift CG-inversion at the precision $\varepsilon / 2$.

The lattice sizes we employed were $4 \times 10^{2} \times 16,6 \times 14^{2} \times 24$ and $8 \times 18^{2} \times 32$, which ensure that the transverse dimensions remains in the confined phase at $2 T_{c}$. The corresponding couplings are respectively the known critical couplings on $N_{t}=8,12$ and 16 lattices. These $\beta$ values are $6.0625,6.3384$ and 6.55 . For the last value, no infinite volume extrapolation was available, unlike the first two. Our choice was motivated by the 2-loop $\beta$-function, and consistency with the the finite volume results. In each case, configurations separated by 1000 sweeps of a Cabbibo-Marinari update were generated and 20-25 such configurations were used to compute the quark propagators. The propagator $G$ was computed on 12 point sources ( 3 colors and 4 spins) for 8 quark masses 
from $m / T=0.008$ to 0.8 using a multi-mass inversion of $D^{\dagger} D$. The tolerance used was $\varepsilon=10^{-3}$ in this outer conjugate gradient.

\section{Results}

Figure 11 displays our results for the correlation functions of $\rho$ and $\pi$ along with the corresponding ideal gas result on the $6 \times 14^{2} \times 24$ lattice (left) and $8 \times 18^{2} \times 32$ lattice (right). Both display an excellent $\cosh$ behaviour, indicative of a dominance of a single mass scale, as the line of a $\cosh$-fit in each case shows. Moreover, one sees a very good agreement of the $\rho$-correlator with the ideal gas on both lattices in essentially its entire 6-7 orders of magnitude fall. There are, however, differences for the pion. In order to exhibit the comparison with ideal gas more clearly,
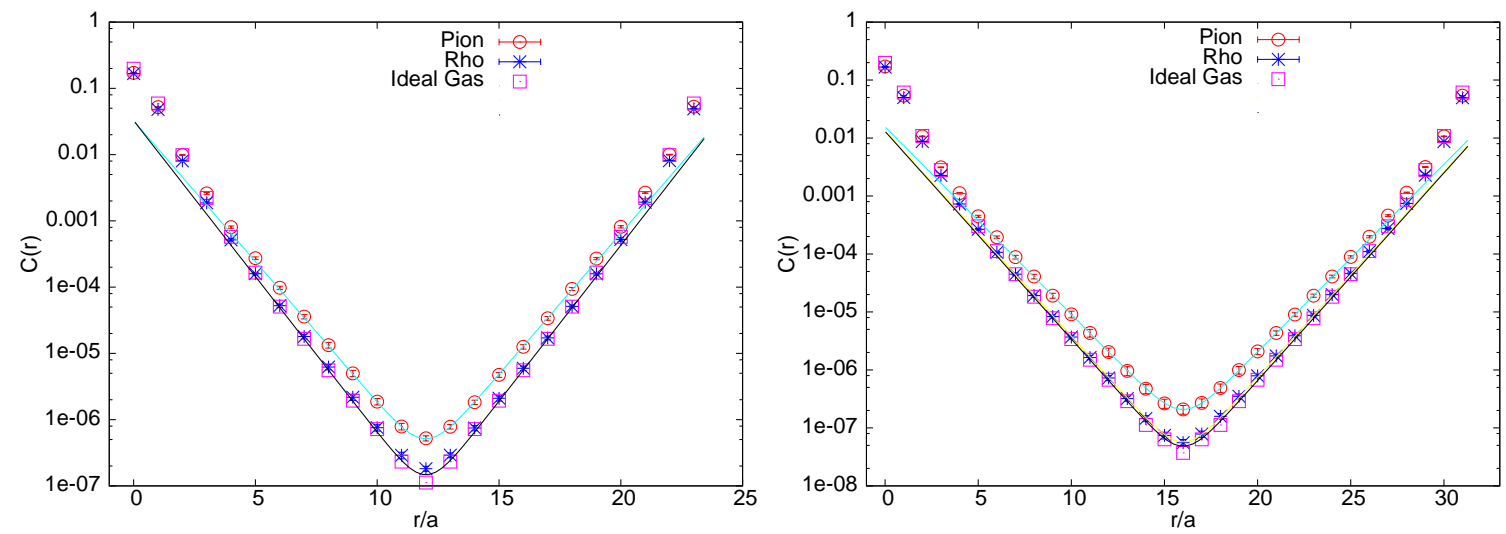

Figure 1: Correlators for pion and rho compared with the corresponding ideal gas results on a $6 \times 14^{2} \times 24$ lattice (left) and a $8 \times 18^{2} \times 32$ lattice (right) at $T=2 T_{c}$. The lines are single $\cosh \mathrm{fi}$ ts.

we show in the left panel of Figure 2 the ratios of the two correlators with that for ideal gas on the our largest lattice. While the deviations from ideal gas seem at $\sim 20 \%$ level for $\rho$, those for $\pi$-correlator range up to a factor of 5-6. Note that in both cases the ideal gas is larger at small distances and smaller at large distances. As the right panel of the Figure 2 shows, the deviations
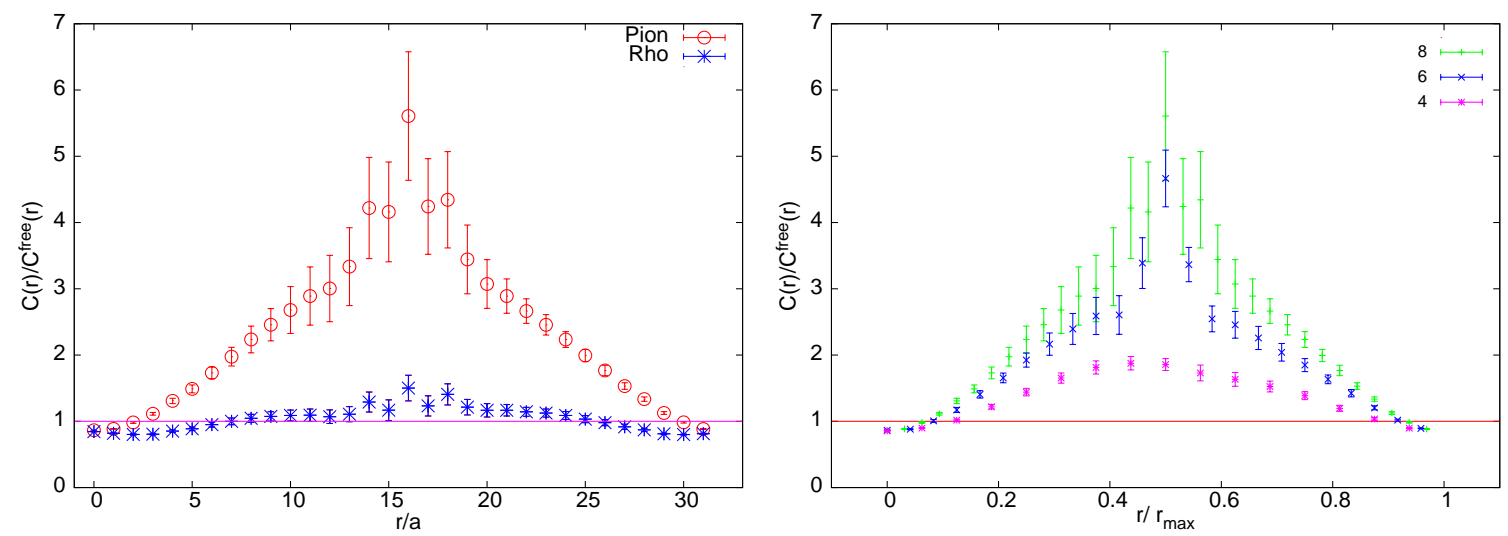

Figure 2: Ratios of pion and rho correlators on $8 \times 18^{2} \times 32$ lattice with the corresponding ideal gas results (left) and the variation of the pion-ratio with $N_{t}$ (right). 
from ideal gas appear to increase progressively in the continuum limit, i.e, with increasing $N_{t}$. Similar trend can also be seen in the case of $\rho$, although the size of deviations and the errorbars make it much less conclusive. We define local masses, as usual, from the ratios of correlation function at successive $r$ values. As may be anticipated from Figure 1, these local masses
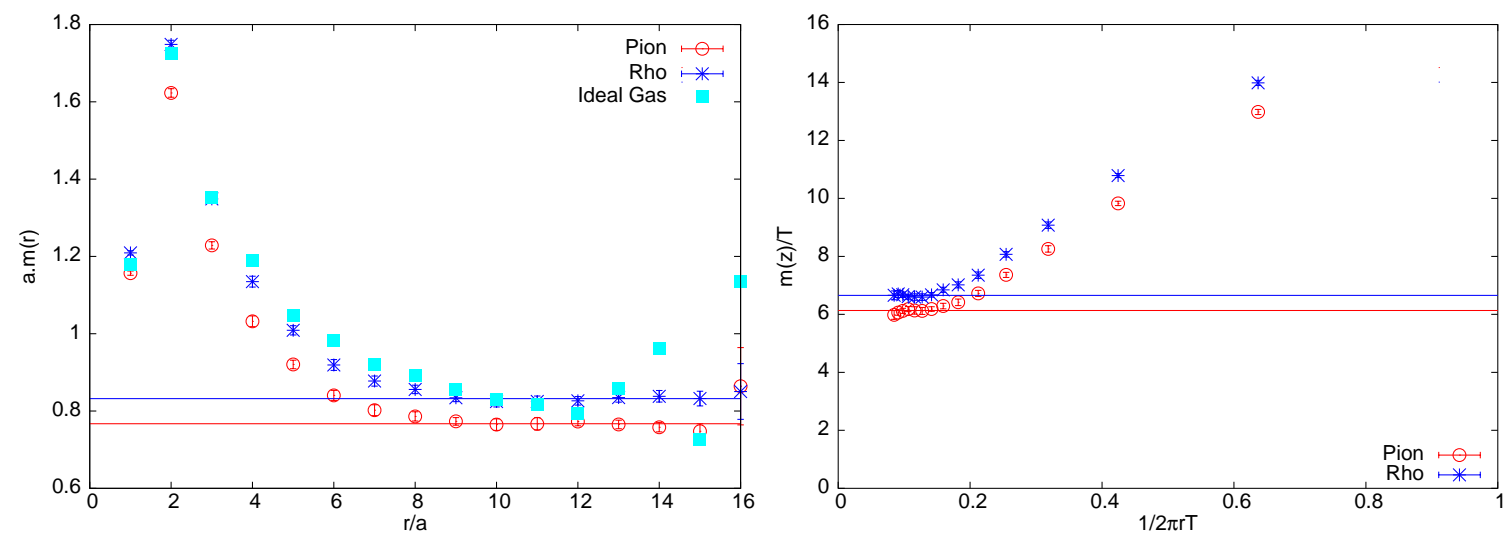

Figure 3: Left panel shows the effective mass as a function of the separation $r$ on $8 \times 18^{2} \times 32$ lattice. The horizontal lines indicate the corresponding mass estimate. The right panel displays the plateau behaviour in another variable advocated in [15].

for both $\rho$ and $\pi$ show demonstrate an excellent plateau behaviour. This is exhibited in the left panel of Figure 3. The ideal gas results are also shown on the same panel for a comparison. One sees very similar behaviour for them as the $\rho$-local mass but for the last three points which have a distinct oscillatory pattern. This should be contrasted with the corresponding results for the staggered fermions [9] or Wilson fermions [15], both of which display rather limited range of plateau, if at all. The right panel shows this explicitly by displaying the local masses as a function of a variable, $1 / 2 \pi r T$, advocated in [15]. While the plateau behaviour is manifestly seen even in this variable in our overlap fermion results in the right panel of Figure 3 , no such plateau is visible in the corresponding figure (Figure 1) of [15].

The plateau in local masses also brings out the complementary nature of the long-range information they contain. The small-distance behaviour of the correlator, and the corresponding local masses, have nothing to do with the plateau value. On the other hand, local quantities, such as the chiral condensate, are related to the integral of the correlator and are thus dominated by the small-distance behaviour only. From Figure 1, one thus surmises that the chiral condensate agrees with its ideal gas value well on both $N_{t}=6$ and 8 , although the local mass for pion in either case does not. Furthermore, the existence of the plateau suggests existence of genuine bound states in both $\rho$ and $\pi$ channels.

Figure 4 exhibits our results on $N_{t}=6$ and 8 , together with our earlier results [11] on $N_{t}=4$ for the ratio of $m_{\pi} / m_{\rho}$ (left) and $m_{\pi} / m_{\text {free }}$ (right). The estimates for $m_{\pi}$ and $m_{\rho}$ were obtained from the plateaux in Figure 3, and checked independently by a direct single cosh fit to the correlation function in the interval of the local mass plateau in each case. The $m_{\text {free }}$ too was obtained from a fit, and was found to agree with the corresponding value of its smaller plateau region. Also shown are our results on cubic lattices having the same transverse size. Both panels display very little, if any, lattice spacing, $a$, dependence, suggesting the pion screening length to remain about $8 \%$ below the 

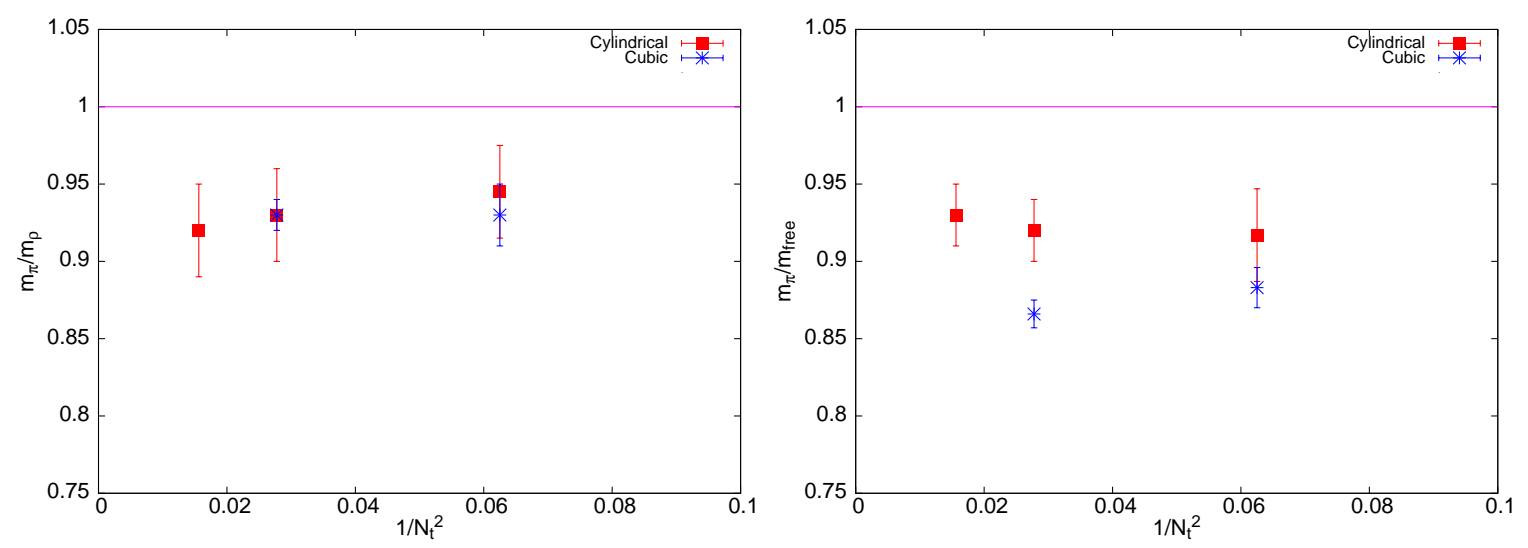

Figure 4: Continuum limit of the ratios $m_{\pi} / m_{\rho}$ (left) and $m_{\pi} / m_{\text {free }}$ (right).

ideal gas value, or more than $2 \sigma$ away, whereas the $m_{\rho}$ is consistent with the ideal gas, as seen by comparison of the two panels or alternatively Figure 3. The ratio $m_{\pi} / m_{\rho}$ does not appear to get affected by the geometry of the lattice, whereas the ratio $m_{\pi} / m_{\text {free }}$ seems smaller on the cubic lattice.

\section{Summary}

Extending our earlier work [11] on the hadronic screening lengths on $N_{t}=4$ lattice to $N_{t}=6$ and 8 at a fixed temperature of $2 T_{c}$ on lattices with large $z$ extent, we found a single $\cosh$ behaviour in both $\rho$ and $\pi$ correlators on both lattices. This led to a convincing plateau behaviour in the corresponding local masses, suggesting the presence of a bound state in each case. The $\rho$-correlator appeared to be in very good agreement with the ideal gas correlator on the same lattice whereas the $\pi$-correlator differed from it on all $N_{t}$. In fact, the deviations appear to increase in the continuum limit, i.e, with increasing $N_{t}$. Ratios of the extracted screening masses displayed very little, if any, dependence on the lattice spacing. While the $\rho$ screening mass is in agreement with the free value, the $\pi$ screening mass remained lower by about $8 \%$ or more than $2 \sigma$. Its constancy with the lattice spacing $a$ suggests the deviation to be a genuine interaction effect.

\section{Acknowledgements}

The computations reported here were performed on the CRAY X1 of the Indian Lattice Gauge Theory Initiative at the Tata Institute of Fundamental Research. It is a pleasure to thank the system administrator, Ajay Salve. The kind hospitality of the Service de Physique Théorique, Saclay, where this manuscript was completed during a visit, is gratefully acknowledged. This work was funded by the Indo-French Centre for the Promotion of Advanced Research under its project number 3104-3.

\section{References}

[1] R. V. Gavai and S. Gupta, Phys. Rev. D73, 014004 (2006). 
[2] C. DeTar and J. Kogut, Phys. Rev. Lett. 59, 399 (1987); Phys. Rev. D 36, 2828 (1987).

[3] K. D. Born et al., Phys. Rev. Lett. 67, 302 (1991).

[4] R. V. Gavai and S. Gupta, Phys. Rev. Lett. 83, 3784 (1999).

[5] A. Gocksch et al., Phys. Lett. B 205, 334 (1988); S. Gupta, Phys. Lett. B 288, 171 (1992).

[6] S. Gottlieb et al., Phys. Rev. Lett. 59, 1881 (1987), Phys. Rev. D 47, 3619 (1993), ibid. D 55, 6852 (1997); J. B. Kogut et al., ibid. D 58, 054504 (1998).

[7] G. Boyd et al., Z. Phys. C 64, 331 (1994); R. V. Gavai and S. Gupta, Phys. Rev. Lett. 85, 2068 (2000).

[8] T. Hashimoto et al., Nucl. Phys. B 400, 267 (1993); Ph. de Forcrand et al., Phys. Rev. D 63, 054501 (2001); E. Laermann and P. Schmidt, Eur. Phys. J. C 20, 541 (2001).

[9] R. V. Gavai and S. Gupta, Phys. Rev. D 67, 034501 (2003).

[10] H. Neuberger and R. Narayanan, Phys. Rev. Lett. 71, 3251 (1993).

[11] R. V. Gavai, S. Gupta and R. Lacaze, Phys. Rev. D 65, 094504 (2002).

[12] H. Neuberger, Phys. Lett. B 417, 141 (1998).

[13] J. van den Eshof et al., Comput. Phys. Comm. 146, 203 (2002).

[14] R. V. Gavai, S. Gupta and R. Lacaze, Comput. Phys. Comm. 154, 143 (2003).

[15] S. Wissel, S. Datta, F. Karsch, E. Laermann and S. Shcheredin, PoS LAT2005, 164 (2005). 